\title{
Personas con discapacidad, salud laboral y capacidades en las leyes del 2011-2020.
}

\author{
People with disabilities, occupational health and capacities in laws from 2011-2020.
} Pessoas com deficiência, saúde do trabalhador e capacidades nas leis de 2011-2020.

\author{
Natalia Eugenia Gómez-Rúa ${ }^{1 *}$ \\ Marisol Moreno-Angarita ${ }^{2}$ \\ María Luisa Toro-Hernández $z^{3}$ \\ Diego Alveiro Restrepo-Ochoa ${ }^{4}$ \\ Diana Carolina Silva-Sánchez ${ }^{5}$
}

\begin{abstract}
Autor de correspondencia
$1^{*}$ Abogada. Especialista en Derecho de la Seguridad Social. Magíster en Derecho de la Universidad CES. Candidata a Doctora en Salud Pública. Coordinadora de Posgrados de la Facultad de Derecho de la Universidad CES. Correo electrónico: ngomez@ces.edu.co (iD)

${ }^{2}$ Fonoaudióloga. Magister en Comunicación. Doctora en Salud Pública. Profesora Asociada de la Universidad Nacional. Correo electrónico: mmorenoa@unal.edu.co iD

${ }^{3}$ Ingeniera Biomédica. Doctora en Rehabilitation Science. Docente Universidad CES. Correo electrónico: mhtoro@ces.edu.co (iD)

${ }^{4}$ Psicólogo. Magister en Psicología. Doctor en Salud Pública. Decano de la Facultad de Psicología de la Universidad CES. Correo electrónico: drestrepo@ces.edu.co (iD)

${ }^{5}$ Enfermera. Especialista en gerencia de salud ocupacional. Magíster en Educación. Estudiante de Doctorado en Salud Pública de la Universidad CES. Correo electrónico:

silva.diana@uces.edu.co iD
\end{abstract}

C) Universidad Francisco de Paula Santander. Este es un artículo bajo la licencia CC-BY-NC-ND

\section{Resumen}

Objetivo: Identificar los conceptos de capacidades en las leyes relacionadas con la salud laboral para las personas con discapacidad en Colombia, entre el 2011 y 2020. Materiales y métodos: Cualitativo con un enfoque metodológico hermenéuticocomprensivo a través de un análisis de contenido. El rastreo de la información se realizó a través de los anales del Congreso de la República que obran en archivos digitales denominados gacetas, producidos desde el año 2011-2020. También se incluyó la Ley 361 de 1997, la cual en materia laboral reguló la estabilidad laboral reforzada. Para el procesamiento de la información se utilizó el programa Atlas/Ti, versión 8. Las categorías de análisis que se tuvieron en consideración fueron: salud, trabajo, empleo, discapacidad, laboral y capacidades. Resultados: El estudio evidenció algunos conceptos de capacidades relacionados con las posibilidades que tienen las personas con discapacidad de elegir funcionamientos valiosos para su vida; sin embargo, las normas siguen presentando vacíos en cuanto a la definición de capacidad y a una orientación hacia los funcionamientos que no permiten a las personas con discapacidad, desarrollar sus capacidades. Conclusiones: Desde la perspectiva de la salud pública, son claras las desigualdades que se pretenden solucionar con normas; pero puede afirmarse que, desde lo legislativo, las capacidades son abordadas desde conceptos muy generales y con elementos básicos. Por otra parte, respecto al desarrollo de capacidades de las personas con discapacidad y la posibilidad de evitar la discriminación de esta población en los entornos laborales, sigue siendo un reto de cara a la práctica y a la inclusión social.

Palabras clave: Salud laboral; trabajadores; salud pública; normas jurídicas ADstract

Objective: Identify the concepts of capacities in laws related to occupational health for people with disabilities in Colombia, between 2011 and 2020. Materials and methods: Qualitative study with hermeneutic-comprehensive methodological ap-

Recibido: 18 noviembre 2019 Aprobado: 25 abril 2020

Para citar este artículo/ To reference this article/ Para citar este artigo/

Gómez-Rúa Natalia Eugenia; Moreno-Angarita Marisol; Toro-Hernández María Luisa; Restrepo-Ochoa Diego Alveiro; Silva Sánchez Diana Carolina. Personas con discapacidad, salud laboral y capacidades en las leyes del 2011-2020. Rev. cienc. cuidad. 2020; 17(2): 116-128. https://doi.org/10.22463/17949831.1850 
proach through the analysis of the content. The tracking of information was made through the annual reports from the Congress of Colombia found in digital archives denominated gazettes, issued between 2011- 2020. Law 361 of 1997, which regulates the reinforced labor stability was also included. For the processing of information, the $8^{\text {th }}$ version of the program Atlas/Ti was used. The categories for analysis that were considered were: health, work, employment, labor disability, and capacities. Results: The study showed some capacity concepts related to the possibilities of people with disabilities of choosing valuable functions for their life: however, laws keep presenting voids regarding the definition of capacity and an orientation towards working operations that do not allow people with disabilities to develop their skills. Conclusions: From the public health standpoint, the inequalities that the laws pretend to solve are clear; but it can be claimed, from a legislative perspective, that the capacities are addressed from very general concepts and with basic elements. On the other hand, regarding the development of skills in people with disabilities and the possibility of avoiding discrimination for this population in work environments, it remains a challenge for practice and social inclusion.

Keywords: Occupational health; workers; public health; legal norms

\begin{abstract}
Resumo
Objetivo: Identificar os conceitos de capacidades nas leis relacionadas com a saúde do trabalhador para as pessoas com deficiência na Colômbia, entre 2011 e 2020. Métodos: Estudo qualitativo com metodologia hermenêutico-compreensiva na análise de conteúdo. A procura da informação realizou-se através dos anais do Congresso da República que repousam em arquivos digitais nomeados "gacetas", produzidos desde 2011 a 2020. Para o processamento da informação empregou-se o software Atlas/Ti, versão 8. As categorias na análise foram: saúde, trabalho, emprego, deficiência laboral e capacidades. Resultados: O estudo evidenciou alguns conceitos de capacidades relacionados com as possibilidades que tem as pessoas com deficiência de escolher funcionamentos de valor para as suas vidas; entretanto, as normas continuam apresentando vazios na definição de capacidade e à orientação aos funcionamentos, impedindo que às pessoas com deficiência desenvolver as suas capacidades. Conclusões: Na perspectiva de saúde pública, são claras as desigualdades que pretendem solucionar com as normas, porém desde a legislação, as capacidades são definidas com conceitos superficiais e básicos. Por outro lado, em relação com desenvolvimento de capacidades nas pessoas com deficiência e a possibilidade de evitar a discriminação e preconceito no ambiente de trabalho, continua sendo um desafio de cara à prática e à inclusão social.
\end{abstract}

Palavras-chave: Saúde do trabalhador; trabalho; saúde pública; normas jurídicas

\section{Introducción}

La Organización Internacional del Trabajo (OIT) ha indicado que en el mundo hay 1000 millones de personas con discapacidad de las cuales al menos 785 millones están en edad de trabajar (1). Por su parte, la Organización Mundial de la Salud (OMS) precisa que tienen peores condiciones de trabajo y salud; se ha reconocido que representan las tasas más bajas de empleo y perciben menos salario (2). Asimismo, el Banco Mundial indica que el Producto Interno Bruto (PIB) mundial pierde entre \$ 1.37 billones de dólares y $\$ 1.94$ billones de dólares debido a la discapacidad (3).

Desde el punto de vista de la salud Pública, el empleo/ trabajo/ocupación son un determinante social de la calidad de vida de las personas en general, y con mayor relevancia de las personas con discapacidad (4); es por eso, que desde esta perspectiva ha sido relevante el análisis de estos factores en el contexto de América Latina y el Caribe. Se afirma que entre el $80 \%$ y el $90 \%$ de las personas con discapacidad están desempleadas (5). En respuesta a esta situación, los países de la región han puesto en marcha diversos mecanismos para fomentar el empleo de este tipo de personas como leyes, normas y programas (6). En el caso de Colombia, solamente se encuentran trabajando el 5,01\% de las personas con discapacidad (7).

Respecto al empleo, es pertinente revisar la existencia de referentes normativos que favorezcan la inclusión laboral de esta población. A este respecto, si se tiene en cuenta que los países miembros de la Organización de Naciones Unidas (ONU) han ratificado la Convención de los Derechos de las Personas con Discapacidad, como Colombia (2011), tienen el deber de insertar en sus propuestas 
$1794-9831$

E-ISSN 2322-7028

Vol. 17 No. 2

May - Ago 2020

Cúcuta, Colombia legislativas el concepto de discapacidad presentado en la Convención (8). Es decir, comprenderla como el resultado de la interacción entre las deficiencias humanas y el medio en el que se desenvuelve la persona $(8,9)$.

De acuerdo con lo expuesto más arriba, es importante precisar que este estudio se realiza a partir de la premisa por la cual la promoción de la salud en el trabajo y la protección de la salud de los trabajadores forman parte de la Salud Pública; por tanto debe ser un objetivo fundamental de las administraciones sanitarias al planificar sus actividades (5). De ahí, que se deba indicar que el modelo de salud laboral da cuenta de distintos niveles causales: el nivel del trabajador (formación, información, equipos de protección individual, etc.); la empresa (cambios organizativos, diseño de herramientas, control de emisiones, etc.); y, los gobiernos (regulación, inspección de trabajo, derechos sociales, etc.). A su vez hay que señalar que estos niveles inciden en la creación de unas condiciones de trabajo, que no supongan riesgos de lesión o enfermedad, sino que representen la dimensión positiva del trabajo para la salud de las personas $(10,11)$.

En el caso de Colombia, existe un marco normativo orientado a dar cumplimiento a la salud laboral; sin embargo, existen condiciones de pobreza, desempleo y deficiencias en las condiciones laborales, que también hacen parte de la realidad de las personas con discapacidad. En este sentido, se puede afirmar que no es suficiente referirse a la inclusión laboral, sino que se debe verificar si los preceptos legales relacionados con la salud laboral, permiten que las personas con discapacidad puedan desarrollar sus capacidades, siempre que cuenten con las condiciones en su entorno, para romper con las barreras respecto a la participación y la igualdad.

Por otra parte, en la teoría de justica de Rawls, se desarrolla el enfoque de capacidades y funcionamientos de Amartya Sen, quien propone una lista heterogénea de capacidades en lugar de una lista de bienes primarios (12). Desde esta óptica, se entiende la libertad como una ventaja individual de la persona para hacer cosas que tenga razón de valorar, diferenciando los funcionamientos y las capacidades. Las capacidades se refieren a funcionamientos valiosos para las personas, no solo en el hacer sino en una elección voluntaria y reivindicatoria de su dignidad como seres humanos (13). Por su parte, los funcionamientos reflejan las diversas cosas que las personas pueden hacer o ser al vivir, entendidos como la situación en que se encuentran respecto a los recursos que adquie- ren por su trabajo o por el intercambio con otros seres humanos (13).

No obstante, el mismo autor, aunque apunta a la relevancia central de la desigualdad de capacidades en la evaluación de las disparidades sociales, no propone ninguna fórmula específica sobre decisiones de políticas. Asimismo, Sen no se refiere específicamente a las personas con discapacidad, solamente explica que presentan una desventaja en términos de libertad para vivir una vida buena, en tanto que la oferta disponible es restringida y escasa (13); pero sí considera importante estudiar seriamente los derechos humanos (13).

A este tema dedica un espacio importante en su obra "La idea de la Justicia", en la que expresamente indica que la idea de derechos humanos es usada para cosas distintas de la producción legislativa. Agrega, que deben entenderse como reivindicaciones éticas constitutivamente vinculadas a la importancia de la libertad humana; por tanto, los derechos son libertades, no solamente individuales sino de los otros, que van más allá de los placeres y la satisfacción de los deseos, en que se concentran los utilitaristas (13).

Sen también indica que cuando las personas adquieren capacidades y alcanzan agencias, entendidas como la habilidad de definir las metas propias de forma autónoma y de actuar a partir de las mismas, supone que se dan en el ejercicio de la libertad de ser agente, es decir, cuando se es libre de hacer y conseguir los propósitos que se consideran importantes. En definitiva, los términos capacidades y agencias son vistos como facetas del bienestar, entendido éste como vectores de realización o capacidad para conseguir realizaciones valiosas (14).

Sobre los postulados anteriores, Nussbaum profundiza respecto al concepto de capacidades y asevera que no solamente se debe tener la posibilidad de elegirlas, sino que hay unas mínimas que se deben ofrecer. Sin dejar de ser una línea del liberalismo igualitarista, en su discurso está explícito lo individual y lo colectivo que deben construirse alrededor de la discapacidad; es decir, que aunque las capacidades están a disposición de la persona, su elección debe estar garantizada por el Estado, entendida como una construcción social y un escenario político (12). Para esta autora, el enfoque de capacidades es una doctrina política acerca de los derechos básicos, ya que al no garantizarlos constituye una violación grave. De igual forma, reflexiona sobre la idea de una vida acorde con la dignidad del ser humano; además, plantea que desde un 
umbral de las capacidades no sólo es posible una vida humana, sino una buena vida, para lo cual se requiere una lista mínima de estas (12).

Algunos autores atribuyen a las capacidades una especial utilidad para la interpretación de la Convención Internacional sobre los Derechos de las Personas con Discapacidad; del mismo modo, resaltan su importancia para mejorar las posibilidades de las personas con discapacidad respecto a sus derechos y dignidad (15-19). Por otro lado, señalan que tienen una relación estrecha con la salud pública, desde su perspectiva de conjunto de acciones individuales y colectivas, públicas y privadas para hacer posible el bienestar y el buen vivir humano (20). En este sentido, que observa a las personas con discapacidad desde sus determinantes sociales, calidad de vida, estilos de vida y en general, el bienestar que se pretende para lograr una sociedad más justa con participación social.

Con relación a lo expuesto anteriormente, en ésta investigación se consideró relevante un análisis conceptual de capacidades en personas con discapacidad en el ámbito legislativo, teniendo en cuenta que las leyes deben ser conocidas por todos los ciudadanos, especialmente cuando se trata de grupos socialmente vulnerables. De la misma manera, deben ser estudiadas de forma interdisciplinar para una aplicación efectiva sobre la población a la que están dirigidas. De esta forma se construirá el conocimiento científico y se aportará al bienestar y salud de los trabajadores con discapacidad.

Por lo tanto, para alcanzar el objetivo del presente estudio, se revisó la producción normativa sobre el eje temático, con posterioridad a la ratificación de la Convención dada en el año 2011, encontrando siete leyes en campos específicos como salud, riesgos laborales y trabajo.

\section{Objetivo}

Identificar los conceptos de capacidades en las leyes relacionadas con la salud laboral para las personas con discapacidad en Colombia, entre el 2011 y 2020

\section{Materiales y métodos}

El enfoque metodológico de la investigación es el hermenéutico-comprensivo. De acuerdo con lo planteado por Gadamer la hermenéutica "es la herramienta de acceso al fenómeno de la comprensión y de la correcta interpretación de lo comprendido (...) comprender e interpretar no es sólo una instancia científica, sino que pertenece con toda evidencia a la experiencia humana en el mundo" (21) como es la producción normativa de la salud de los trabajadores con discapacidad. El objeto de análisis para la construcción de capacidades, está planteado desde la personas con discapacidad, como sujetos no como objetos, donde los individuos se reconocen como personas, porque a partir de esta perspectiva aprenden a referirse a sí mismos y a los otros, como seres a los que se les atribuyen cualidades o capacidades positivas (22). Igualmente, se enmarca bajo supuestos éticos con el abordaje de la salud laboral y al versar concretamente, sobre las personas con discapacidad, se encuentra fundamentada en la teoría de derechos. En este sentido, hay que aclarar que las capacidades se encuentran íntimamente relacionadas con el concepto de dignidad humana, para dar respuesta no solo a posturas individuales, sino que favorezcan una ética colectiva de la discapacidad en ambientes de trabajo. Este proyecto de investigación obtuvo el aval del Comité Institucional de Ética en Humanos, de la Universidad CES a través del acta 87, del 9 de noviembre de 2016.

El rastreo de la información se realizó a través de los anales del Congreso de la República que obran en archivos digitales denominados gacetas, producidos desde el año 2011-2020. También se incluyó la Ley 361 de 1997, la cual específicamente, en materia laboral regula la estabilidad laboral reforzada (hoy ocupacional) con la finalidad de que ninguna persona con discapacidad física, síquica o sensorial sea despedida por esta razón y sin autorización de la autoridad administrativa correspondiente (el inspector de trabajo). Con esta Ley se garantiza la no discriminación, concepto referenciado en la Convención de los Derechos de las Personas con Discapacidad. A continuación, se describen los resultados de la búsqueda. Tabla 1 . 
ISSN-PRINT

$1794-9831$

E-ISSN 2322-7028

Vol. 17 No. 2

May - Ago 2020

Cúcuta, Colombia
Tabla 1. Leyes relacionadas con el enfoque de derechos de la salud laboral de las personas con discapacidad en Colombia.

\begin{tabular}{|c|c|c|c|}
\hline Ley & Año & Tema & Subtema de la salud laboral \\
\hline 361(23) & 1997 & $\begin{array}{l}\text { Mecanismos de integración social de } \\
\text { las personas con limitación }\end{array}$ & $\begin{array}{l}\text { Aspectos referidos a los gobiernos: relacionados con estructura } \\
\text { productiva, relaciones laborales y mercado de trabajo. }\end{array}$ \\
\hline 1438 & 2011 & $\begin{array}{l}\text { Reforma al Sistema General de } \\
\text { Seguridad Social en Salud }\end{array}$ & $\begin{array}{l}\text { Aspectos referidos a los gobiernos: relacionados con estructura } \\
\text { productiva, relaciones laborales y mercado de trabajo. }\end{array}$ \\
\hline $1450(24)$ & 2011 & $\begin{array}{l}\text { Plan Nacional de Desarrollo 2010- } \\
2014\end{array}$ & $\begin{array}{l}\text { Aspectos referidos a los gobiernos: relacionados con estructura } \\
\text { productiva, relaciones laborales y mercado de trabajo. }\end{array}$ \\
\hline $1562(25)$ & 2012 & Riesgos laborales & Aspectos referidos a las empresas: condiciones de trabajo y empleo. \\
\hline $1566(26)$ & 2012 & $\begin{array}{l}\text { Atención integral a personas que } \\
\text { consumen sustancias psicoactivas }\end{array}$ & $\begin{array}{l}\text { Aspectos referidos a las empresas: condiciones de trabajo y empleo. } \\
\text { Aspectos referidos a los trabajadores: condiciones individuales. }\end{array}$ \\
\hline $1616(27)$ & 2013 & Salud mental & $\begin{array}{l}\text { Aspectos referidos a las empresas: condiciones de trabajo y empleo. } \\
\text { Aspectos referidos a los trabajadores: condiciones individuales. }\end{array}$ \\
\hline $1618(28)$ & 2013 & $\begin{array}{l}\text { Pleno ejercicio de las personas con } \\
\text { discapacidad }\end{array}$ & $\begin{array}{l}\text { Aspectos referidos a los gobiernos: relacionados con estructura } \\
\text { productiva, relaciones laborales y mercado de trabajo. Aspectos } \\
\text { referidos a las empresas: condiciones de trabajo y empleo. Aspectos } \\
\text { referidos a los trabajadores: condiciones individuales. }\end{array}$ \\
\hline 1751(29) & 2015 & Derecho fundamental a la salud & $\begin{array}{l}\text { Aspectos referidos a los gobiernos: relacionados con estructura } \\
\text { productiva, relaciones laborales y mercado de trabajo. Aspectos } \\
\text { referidos a los trabajadores: condiciones individuales. }\end{array}$ \\
\hline $1753(30)$ & 2015 & $\begin{array}{l}\text { Plan Nacional de Desarrollo 2014- } \\
2018\end{array}$ & $\begin{array}{l}\text { Aspectos referidos a los gobiernos: relacionados con estructura } \\
\text { productiva, relaciones laborales y mercado de trabajo. }\end{array}$ \\
\hline
\end{tabular}

Fuente: construcción propia de de los autores

Como se puede observar, se incluyó solamente en las leyes lo relacionado con: los conceptos, las comprensiones y las conceptualizaciones relacionadas con la salud laboral, que aparecen esbozadas en las normas expedidas por el Congreso de la República; es decir, que se excluyeron los decretos, las resoluciones y las circulares, que responden más a la aplicación de las leyes. Luego, se realizó un análisis de contenido que según Vásquez et al., "se caracteriza por la clasificación sistemática, descripción e interpretación de los contenidos del documento, que se llevará a cabo de acuerdo con los objetivos del estudio" (31). Por su parte, Páramo señala que este tipo de análisis "consiste en identificar dentro de un todo (el documento obtenido a partir de determinada fuente) los datos y clasificarlos con base en las diferentes categorías de análisis (esto demanda un ejercicio semántico); describir los contenidos a los que se refieren esos datos e interpretar esos contenidos de acuerdo con los objetivos que se pretendan, se trata pues de investigar el significado simbólico de los mensajes" (32). Se utilizó el programa informático Atlas/Ti, versión 8. Las categorías de análisis que se tuvieron en consideración fueron: salud, trabajo, empleo, discapacidad, laboral y capacidades. A continuación, se presentan los principales resultados y las discusiones generadas del análisis de contenido de las citadas leyes.

\section{Resultados}

Los resultados del análisis evidenciaron concretamente conceptos sobre el término capacidades y discapacidad mental como se expone a continuación:

\section{Términos relacionados con las capacidades}

Sobre los términos relacionados con las capacidades en las leyes, que tienen que ver con la salud de las personas con discapacidad, se reflejan en la discusión entre funcionamientos y capacidades planteadas por Sen y Nussbaum, las cuáles se describen en la Figura 1. 


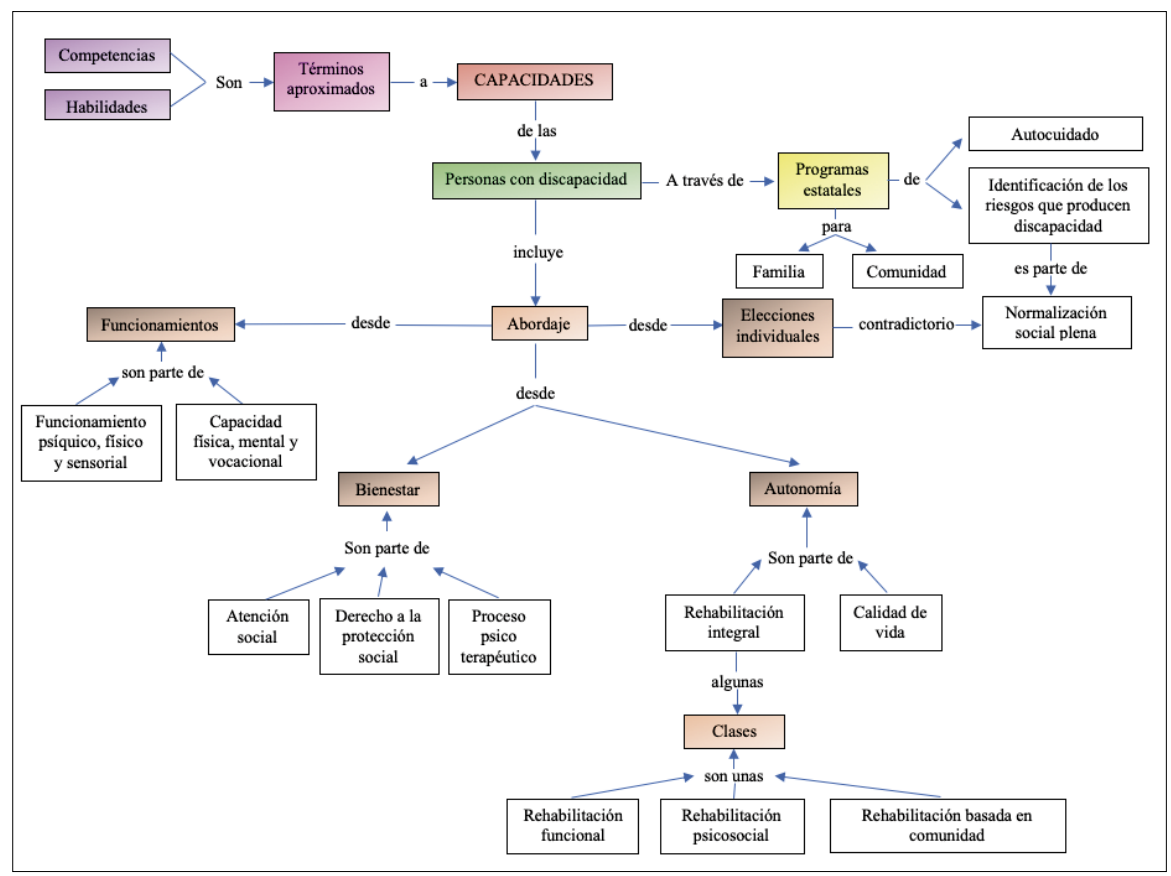

E-ISSN 2322-7028

Vol. 17 No. 2

May - Ago 2020

Cúcuta, Colombia

Figura 1. Red de capacidades

Fuente: Construcción propia de los autores

De acuerdo con la Figura 1, los términos aproximados a las capacidades en el contexto de la salud laboral son las competencias y las habilidades que teóricamente se orientan a los funcionamientos. De otra parte, el abordaje de la discapacidad en el trabajo se concreta en cuatro conceptos: funcionamientos, bienestar, autonomía y elecciones individuales. Respecto a los funcionamientos éstos se apoyan en lo psíquico, lo físico, lo mental o lo vocacional. En relación con el bienestar se conjugan la atención social, el derecho a la protección social y los procesos psicoterapéuticos a los que deben tener acceso. Por su parte, la autonomía comprende los derechos a la rehabilitación integral y la calidad de vida. Las elecciones individuales que están en contradicción con la normalización social plena, se vinculan con la presencia de un modelo médico rehabilitador, a través de una norma- tiva que pretende armonizar la Convención de los Derechos de las Personas con Discapacidad en la legislación interna. Sin embargo, se debe resaltar que hay un avance significativo desde una perspectiva del modelo social, ya que hace referencia a las normas y a los programas estatales que involucran a la familia y a la comunidad, con perspectivas interesantes como el autocuidado y la identificación de riesgos que producen discapacidad.

\section{Salud mental, discapacidad y capacidades}

La salud mental surge en el análisis de contenido como una categoría emergente, frente al predominio en las normas de salud laboral de aspectos relacionados con la población con discapacidad mental y sus capacidades; es a partir de esta relación que surge en la normatividad de salud laboral la siguiente red. Ver Figura 2. 
ISSN-PRINT

1794-9831

E-ISSN 2322-7028

Vol. 17 No. 2

May - Ago 2020

Cúcuta, Colombia

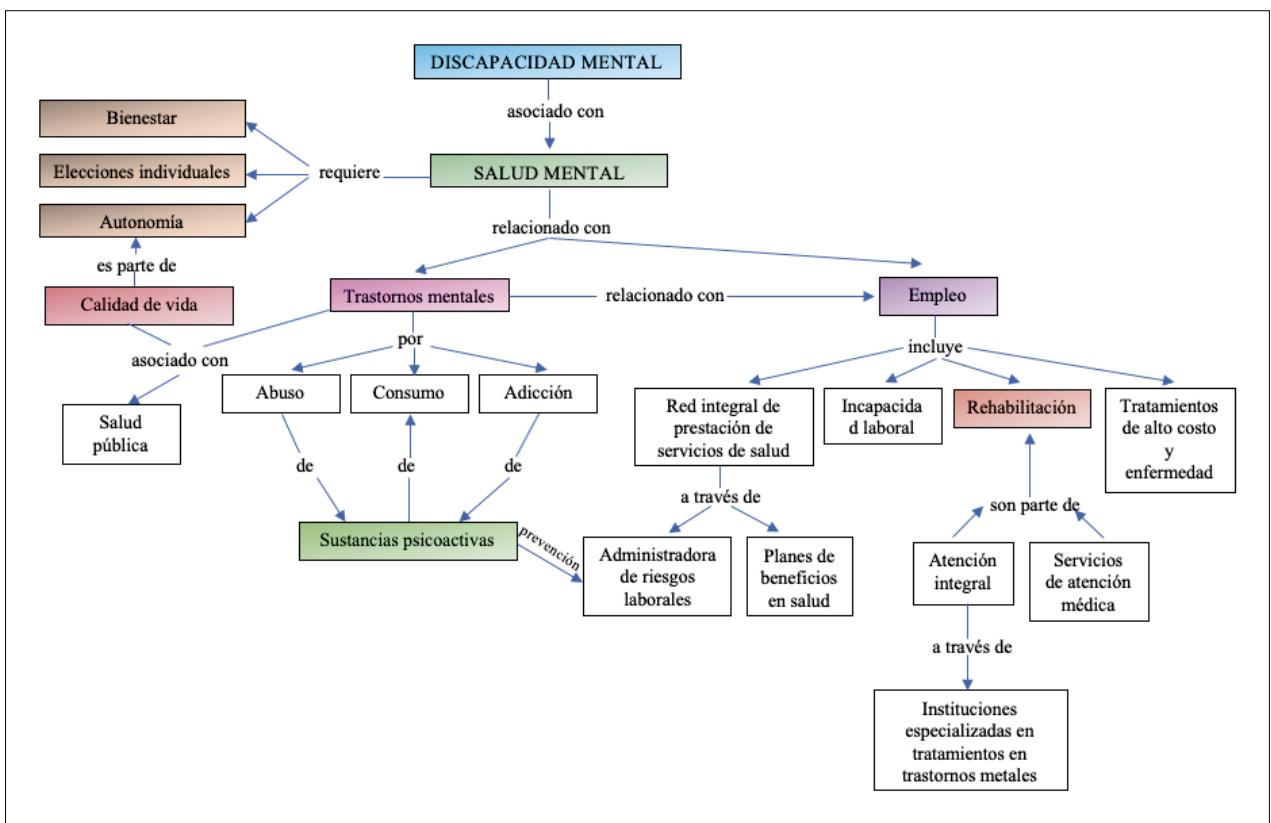

Figura 2. Red de Salud Mental

Fuente: Construcción propia de de los autores

Como puede observarse en la Figura 2, se asocia la discapacidad con la salud mental ocasionada por trastornos mentales relacionados con el consumo de sustancias psicoactivas; por tal motivo se requiere garantizar a la persona con discapacidad que tenga acceso al bienestar y a la posibilidad de realizar elecciones individuales; también, se debe brindarle autonomía y calidad de vida desde una perspectiva de la salud pública. En materia de empleo concretamente, se resalta la red integral de prestación de servicios de salud a través de las administradoras de riesgos laborales y las EPS con sus planes de beneficios; asimismo, se insiste en la necesidad de respetar sus incapacidades médicas y el proceso de su rehabilitación integral, así como los tratamientos de alto costo y las enfermedades laborales que se deriven de sus condiciones de salud.

\section{Discusión}

Respecto a los términos aproximados a capacidades como competencias y habilidades, el artículo 8 de La Ley 1616 explica que las competencias hacen parte de la rehabilitación psicosocial de trastornos mentales y sirven para la mejoría de la competencia individual (27). De manera específica el literal $g$ del numeral 3 del artículo 13 de la Ley 1618 se refiere a las competencias laborales y el numeral 7 del artículo 16 a las competencias comunicativas. La primera tiene como objetivo lograr la certificación en el campo laboral y la segunda, presenta un programa de capacitación en tecnologías de la información y las comunicaciones para personas con discapacidad sensorial y con deficiencias específicas que alteren las competencias para comunicarse a través del lenguaje verbal (28). Como puede verse las competencias se orientan al desempeño y a la productividad, mientras que Nussbaum considera que las capacidades no exigen ganarse el respeto de los demás siendo productivos, sino que deben estar basadas en la dignidad misma de las necesidades humanas y reconoce que la productividad no es la finalidad principal de la vida social (12). En esta medida, el desempeño y la productividad están vinculados a las necesidades de los sectores productivos y a la oferta, por tanto es lo que se ofrece a las personas con discapacidad, sin tener posibilidad de elección.

En cuanto a las habilidades, el artículo 17 de la Ley 1438 explica la atención preferente con fines rehabilitadores (33) y el artículo 18 de la Ley 1618, las habilidades de las personas con discapacidad de acuerdo con el ciclo de la vida (28). Aunque tampoco es un término sinónimo de capacidades, el desarrollo de las habilidades, independiente de las básicas que se deben brindar como lo explica Nussbaum, han sido utilizadas por algunos autores en el abordaje de las capacidades en personas con discapacidad. En tal sentido, Hammell en el marco de terapias ocupacionales las identifica como estrategias ante las desigualdades de oportunidad ocupacional e inequidades en la participación (34) y Moreno et al., en la creación 
de una herramienta denominada Valoración Ocupacional de las Personas con Discapacidad en Colombia (4). En ambos casos pasa lo mismo que con las normas, no se realiza un planteamiento sobre la vida que quieren vivir las personas con discapacidad; es decir, que están más orientadas a los funcionamientos que a las capacidades. Sin embargo, se considera una herramienta valiosa para el desarrollo de las capacidades en el campo laboral, de tal forma que se construya una herramienta pensada para los roles que puede desempeñar en el trabajo cualquier persona con discapacidad.

En las leyes se encuentran abordajes sobre funcionamientos, bienestar, autonomía y elecciones individuales, relacionados con la perspectiva de Sen y Nussbaum sobre las capacidades de las personas con discapacidad. Respecto a los funcionamientos, el artículo 8 de la Ley 361 establece que las personas con discapacidad "que no hayan desarrollado al máximo sus capacidades, o que con posterioridad a su escolarización hubieren sufrido la limitación, tendrán derecho a seguir el proceso requerido para alcanzar sus óptimos niveles de funcionamiento psíquico, físico, fisiológico, ocupacional y social" (23). Por otro lado, la Ley 1618 en su artículo 9 se refiere a la capacidad física, mental y vocacional como parte de la rehabilitación integral (28). Aunque las normas se fundamentan en una visión rehabilitadora que se asocia con las deficiencias y la productividad, el reconocimiento de lo funcional respecto a las capacidades no deja de ser un elemento interesante para cualquier individuo, considerando que la parte biológica que causa la discapacidad es un elemento inequívoco.

Por su parte, el bienestar, entendido desde la perspectiva de Sen, como el conjunto de vectores o elementos de realización y de la capacidad para conseguirlos (14), se asocia en las leyes estudiadas con la atención social presentada a través de la ley 361 (23) y el derecho a la protección social descrito en la Ley 1618, a partir de programas de bienestar para la inclusión de las personas con discapacidad, la promoción de sus derechos y los mecanismos de seguimiento (28). También, de forma muy concreta con los procesos psicoterapéuticos planteados respecto a la salud mental a partir de la Ley 1616 (27). En materia de capacidades el término es relevante, en cuanto a que la persona con discapacidad tenga la libertad de conseguir aquellas cosas que constituyen su propio bienestar, como lo indica Sen (35), a partir de provisiones públicas para la seguridad social o la planificación de la satisfacción de las necesidades básicas (14), como lo pretenden las normas citadas al utilizar este concepto.
Adicionalmente, algunas normas van más allá del bienestar y abordan la autonomía, que en palabras de Sen se refiere al procedimiento de decidir libremente por uno mismo. La Ley 1616 en el numeral 8 del artículo 5 trata la autonomía como un acción intersectorial para lograr la calidad de vida (27) y la Ley 1618 respecto a la rehabilitación integral, la considera como un mecanismo que permite la máxima autonomía e independencia (28). Al respecto, el artículo 8 de Ley 1618, precisa que la rehabilitación integral pretende el "mejoramiento de la calidad de vida y la plena integración de la persona con discapacidad al medio familiar, social y ocupacional, a través de procesos terapéuticos, educativos y formativos que se brindan acorde al tipo de discapacidad". Además, el artículo 9 reconoce el derecho a la rehabilitación con el objetivo de lograr y mantener la máxima autonomía e independencia en su capacidad física, mental y vocacional, así como la inclusión y participación plena en todos los aspectos de la vida (28).

Un aspecto a resaltar es la mención de algunas clases de rehabilitación integral en las últimas leyes proferidas en materia de salud laboral, de forma expresa sobre la rehabilitación funcional. En este sentido el artículo 6 de la Ley 1618, se refiere al proceso de acciones médicas y terapéuticas, encaminadas a lograr que las personas con discapacidad estén en condiciones de alcanzar y mantener un estado funcional óptimo, desde el punto de vista físico, sensorial, intelectual, psíquico o social, de manera que les posibilite modificar su propia vida y ser más independientes (28). En la Ley 1616, artículo 8, se explica la rehabilitación psicosocial como un proceso que facilita la oportunidad a los individuos con un trastorno mental a un óptimo funcionamiento y minimización de discapacidad (27). De modo similar, la Ley 1618, artículo 8, se refiere a la rehabilitación basada en la comunidad para las personas con discapacidad, mediante la integración de las familias y la comunidad en todos los campos de la actividad humana (28). Como puede observarse en su integridad, la rehabilitación incluye los aspectos biológicos y sociales del concepto de discapacidad para lograr la autonomía de las personas. De cualquier manera, es importante dejar sentado que la rehabilitación está más orientada hacia la integración, las capacidades y la inclusión social, específicamente en cuanto a disponer de autonomía para elegir la vida que se quiere vivir. Desde luego no es satisfactoria la rehabilitación integral, por lo tanto las leyes pueden limitar, como indica Sen "el proceso de elección autónoma de la persona respecto a las oportunidades reales de conseguir sus propios objetivos" (13). 
Por otra parte, otras normas profundizan, en el contexto de las capacidades, sobre la posibilidad de elecciones individuales. A este respecto, en el artículo 8 de la Ley 1616, expresamente se indica que la rehabilitación psicosocial pretende potenciar las elecciones individuales para vivir satisfactoriamente en la comunidad (27). La inclusión del término elecciones individuales, así sea en una sola norma, es importante, porque se van abandonando conceptos contrarios a la autonomía y el desarrollo de capacidades de esta población, como la normalización social plena consagrada en el artículo 3 de la Ley 361 (23) y propia de la visión rehabilitadora, que sin lugar a dudas se queda en el ámbito del funcionamiento.

Desde otra perspectiva, en un análisis específico sobre las acciones para el desarrollo de capacidades relacionadas con las personas con discapacidad, se encuentra en el numeral 1 del artículo 8 de la Ley 1618, que se consagran los programas estatales, por cuanto "las entidades nacionales, departamentales, municipales, distritales y locales competentes, así como el Instituto Colombiano de Bienestar Familiar (ICBF), deberán apoyar programas orientados a desarrollar las capacidades de las personas, la familia y la comunidad en el autocuidado y en la identificación de los riesgos que producen discapacidad" (28). Aquí se incluye la familia, entendiendo que son los cuidadores y representantes legales corresponsables del desarrollo de estos programas para los menores de 18-25 años, según el caso.

A partir del planteamiento anterior, a pesar de referirse a capacidades, el discurso se enfoca en la parte de los funcionamientos; y, aunque se reconoce la importancia del papel del Estado para crear programas orientados a su desarrollo, no solo para la persona con discapacidad sino para su familia y la comunidad, solamente se plantean el autocuidado y la identificación de riesgos que producen la discapacidad (este último término utilizado en la Ley 361 como parte de la normalización social plena de la población con discapacidad). A lo que hay que agregar que es importante pensar en una integración normativa que dé mayor importancia a la vida que cada persona con discapacidad quiere vivir. Esta priorización sobre el funcionamiento y la habilitación hacen que todavía varias respuestas programáticas se centren en que la persona debe alcanzar un funcionamiento estándar, desconociendo su individualización.

De igual forma, sobre los términos relacionados con las capacidades, lo que se evidencia en el análisis de las leyes de la salud laboral, es que la norma que tiene mayores abordajes cercarnos a las capacidades es la Ley 1616 referida a salud mental. Como puede observarse en lo expuesto anteriormente se refiere al bienestar, la autonomía y las elecciones individuales de forma expresa estableciendo una relación directa con el desarrollo de capacidades de las personas con discapacidad, posiblemente porque la salud mental ha sido abordada como un tema de salud pública, por su impacto y efectos incapacitantes en la población (36-39). Específicamente, Restrepo et al., se refieren a los estudios realizados sobre los trastornos mentales, a la carga global de la enfermedad en el mundo, a los requerimientos de organismos multinacionales para que se integre la salud mental a los aspectos de salud, a la política social y a las investigaciones sobre este tema (38).

Desde otro punto de vista, en relación con las capacidades, la OMS ha señalado que la salud mental es un aspecto esencial de bienestar en el desarrollo de los individuos (40). Sobre el enfoque de los derechos, la OPS ha reiterado que desde su constitución ha velado porque el goce del grado máximo de la salud no sea solamente un estado o condición de la persona, sino también uno de los derechos fundamentales de todo ser humano (41). Hay que precisar que la discapacidad, se manifiesta en una persona afectada en su salud mental, quien puede presentar una deficiencia psíquica (27) y, a raíz de esta, se le presentan barreras para la participación y la igualdad. Por su parte, la Ley 1616 señala que la discapacidad mental se manifiesta cuando: "una persona padece limitaciones psíquicas o de comportamiento, que no le permiten en múltiples ocasiones comprender el alcance de sus actos; tiene dificultad para ejecutar acciones o tareas y para participar en situaciones vitales. La discapacidad mental de un individuo puede presentarse de manera transitoria o permanente, la cual es definida bajo criterios clínicos del equipo médico tratante" (27).

El lenguaje identificado en la norma respecto a la asociación de la salud mental con la discapacidad mental es positivo, estableciendo una relación con las capacidades, especialmente al plantear que para la salud mental se requiere: bienestar, la posibilidad de elecciones individuales y el ejercicio de la autonomía; ésta incluye, como se indicó anteriormente la calidad vida, tema fundamental para la salud pública en aras del bienestar y salud de las poblaciones.

De otro lado, en la norma la salud mental está relacionada con la norma sobre sustancias psicoactivas, como se evidencia en el artículo 1 de la Ley 1566 en donde se 
asocia el trastorno mental con el consumo, abuso y adicción; además, se lo identifica como un problema de salud pública (26). Situación que desde el campo del conocimiento se ha indicado que incide en la discapacidad, en muertes prematuras, en morbilidad evitable y en lesiones intencionales y accidentales (7). En el ámbito laboral es interesante observar cómo esta Ley se plantea en términos de prevención. Es por eso, que en el artículo 7 se especifica que este aspecto involucra a las administradoras de riesgos laborales a través de los programas de salud ocupacional, hoy de seguridad y salud en el trabajo (6). Esto con el objetivo de garantizar el bienestar de los trabajadores en el marco de la normatividad vigente en Colombia, en materia de riesgos laborales, con la que se pretende no solamente reconocer prestaciones económicas, sino también prevenir las enfermedades laborales, concretamente las Leyes que se refieren a la salud mental.

En el marco del desarrollo de capacidades no se puede desconocer que los casos de discapacidades mentales graves son más complejos y en palabras de Nussbaum requieren una buena asistencia por parte del Estado, que se centrará en el afecto, el equilibrio emocional y la salud (12). Sin embargo, se definen algunos funcionamientos valiosos, pero que difícilmente permitirán que la persona pueda ser libre para hacer y conseguir sus propósitos; aspectos que también han sido planteados por autores como Toboso, Mitra, Reicher, Burchardt, entre otros (13).

Adicionalmente, las Leyes 1566 y 1616, se relacionan con el empleo. El artículo 12 de la ley 1616 incluye dentro de la red prestadora de servicios de salud, los encargados de los planes de beneficio y las administradoras de riesgos laborales (27); es decir, que no solamente participan en la prevención de los riesgos, lo que garantiza una atención integral en materia de salud de los trabajadores con discapacidad mental. Por otra parte, el numeral $7 \mathrm{del}$ artículo 6 se refiere al derecho a aceptar la incapacidad laboral en los términos y condiciones dispuestas por el profesional de salud tratante, garantizando la recuperación en la salud de la persona. Este es un tema relevante, ya que las personas con discapacidad mental pueden sentir temor de enfrentarse al empleador, actitud a la que deben hacer frente mediante el uso de su derecho a la citada incapacidad médica, especialmente porque pueden ser víctimas de la discriminación y las desigualdades a las que son expuestos en el campo laboral, como lo advirtió la OMS en el informe mundial sobre discapacidad (39). Desde esta perspectiva, el empleador debe ser el actor principal en el contexto de la salud laboral para garantizar los derechos de los trabajadores respecto a su salud, puesto que es un estado de bienestar al que todos tiene derecho.

Por último, respecto al empleo también se debe incluir la rehabilitación y los tratamientos de alto costo y enfermedades laborales que cita la Ley 1618. En tal sentido, en el numeral 8 del artículo 5 se expone el tema de la rehabilitación para la integración en la comunidad, lo cual implica la atención integral, a través de instituciones especializadas en tratamientos de trastornos mentales y de atención médica en general. Sobre los tratamientos de alto costo y enfermedades laborales que cita el artículo 40 es muy importante, ya que se tiene en cuenta el que se incluyan las patologías y niveles de deterioro de la salud mental, especialmente las causadas por exposición a factores de riesgo psicosociales en el trabajo (12). De las evidencias anteriores, se puede inferir que estas posturas normativas implican un compromiso por parte del Estado y de los empleadores frente a las personas con discapacidad, con el fin de lograr una efectiva inclusión laboral.

\section{Conclusiones}

Respecto a las capacidades, su abordaje en la ley 361 se orienta más hacia el tema del funcionamiento, debido al predominio de una visión rehabilitadora vigente para la época; además, refleja las concepciones que se tenían acerca de las personas con discapacidad. De otra parte, el tema de las normas sobre la salud laboral, de mayor significado para el debate y el desarrollo de las capacidades de las personas con discapacidad se refleja en las leyes 1566, 16161618 y 1618. En tal sentido, las dos primeras leyes se refieren a los procesos de salud-enfermedad por discapacidad mental. Este tema sobre las capacidades es relevante ya que puede generar funcionamientos valiosos respecto a la vida que desean vivir las personas con discapacidad, para hacer frente a las barreras que les impone la sociedad para desarrollar sus capacidades y adaptarse a lo que les ofrece el mundo del trabajo.

No obstante, sin cuestionar la relevancia que se ha dado en el país a la discapacidad mental, debe advertirse que el desarrollo de capacidades, desde un enfoque de derechos, es para todas las personas con discapacidad, sin segregarlas, un problema al que tienen que hacer adaptarse por las barreras para la participación y la igualdad; de ahí, que sea necesario evitar que se perpetúen, promoviendo verdaderos cambios desde el ámbito normativo.

Es conveniente resaltar que la atención integral por medio de diversas instituciones y equipos de trabajo multi- 
disciplinarios con la participación de un grupo de médicos, optometristas, odontólogos, enfermeros y terapeutas físicos entre otros, es clave para intervenir en las necesidades de la salud física de las personas con discapacidad.

Por último, desde la perspectiva de la salud pública, son claras las desigualdades que se intentan solucionar por medio de las normas; sin embargo, desde lo legislativo, el desarrollo de las capacidades es abordado de forma ge- neral, sin tener en cuenta su aplicación en materia laboral, razón por la cual es imposible evitar la discriminación de esta población en los entornos laborales, convirtiéndose en un reto de cara a la práctica y la inclusión social. En definitiva, estos retos deberán ser implementados mediante programas individualizados, con ajustes razonables, acomodaciones y apoyos idóneos que favorezcan la participación plena.

\section{Referencias bibliográficas}

1. Organización Internacional del Trabajo. Estrategia y plan de acción para la inclusión de la discapacidad 2014-17: un doble enfoque de acciones transversales y específicas para las personas con discapacidad [Internet]. 1a ed. Ginebra: OIT; 2015. 29 p. Disponible en: http://www.ilo.org/wcmsp5/groups/ public/---ed_emp/---ifp_skills/documents/genericdocument/wcms 370773.pdf

2. Navarro JL, Guerrero I, Viana RA. Economía laboral y discapacidad: reflexiones en torno a un asunto de salud pública. MedUNAB [Internet]. 2015 [citado 24 de julio de 2016];18:71. Disponible en: http:// search.ebscohost.com/login.aspx?direct $=$ true\&site $=$ eds-live $\& \mathrm{db}=\mathrm{a} 9 \mathrm{~h} \& A N=116830816$

3. Organización Mundial de la Salud. Plan de acción mundial de la Organización Mundial de la Salud sobre discapacidad 2014-2021: mejor salud para todas las personas con discapacidad. [Internet]. Ginebra: Organización Mundial de la Salud; 2014 [citado 12 de abril de 2015] p. 23. Report No.: A67. Disponible en: http://www.who.int/disabilities/policies/actionplan/disability_action_plan_es.pdf

4. Moreno M, Cortés E, Cárdenas A, Giraldo Z, Mena L. Valoración ocupacional de las personas con discapacidad, desde la perspectiva de las capacidades humanas. Rev Salud Pública [Internet]. 2013 [citado 13 de octubre de 2017];15(5). Disponible en: http://www.redalyc.org/html/422/42230941010/

5. Berman R. Desarrollo Inclusivo: un aporte universal desde la discapacidad. In Discurso pronunciado durante el Primer Congreso Interamericano y Tercer Seminario Nacional sobre Discapacidad y Derechos Humanos: Desafío para el Desarrollo Social Inclusivo [Internet]. Panamá: Banco Mundial; 2005 [citado 5 de octubre de 2016] p. 2-9. Disponible en: http://ecaths1.s3.amazonaws.com/orghosp/901049776

6. Comisión Económica para América Latina y el Caribe. Brechas,ejes y desafios en el vinculo entre lo social y lo productivo [Internet]. Montevideo: CEPAL; 182d. C. [citado 18 de noviembre de 2018]. Disponible en: https://www.cepal.org/sites/default/files/events/files/brechas_ejes_y desafios en_el vinculo_entre lo_social_y lo_productivo.pdf

7. Ministerio de Salud y Protección Social. Observatorio Nacional de Discapacidad [Internet]. [citado 20 de julio de 2016]. Disponible en: http://ondiscapacidad.minsalud.gov.co/Paginas/Home.aspx

8. Organización de Naciones Unidas. Convención sobre los derechos de las personas con discapacidad [Internet]. 2006 p. 35. Disponible en: http://joomla.corteidh.or.cr:8080/joomla/images/stories/Observaciones/2/Anexo\%20XVII/L-8661\%20convenci $\%$ C3\%B3n $\% 20 \mathrm{~d} \% 20 \mathrm{pe} \% 20$ con $\% 20 \mathrm{discapa}$.doc

9. World Health Organization. International classification of functioning, disability, and health: ICF [Internet]. Geneva: OMS; 2001. 228 p. Disponible en: http://apps.who.int/iris/bitstream/10665/43360/1/9241545445_spa.pdf

10. García M. La salud laboral y la salud pública: 10 años de políticas sanitarias de salud laboral. Arch Prev Riesgos Labor [Internet]. 2005 [citado 6 de septiembre de 2015];8:100-2. Disponible en: http:// www.scsmt.cat/Upload/TextComplet/1/0/109.pdf

11. Benavídez F, Boix P, Rodrigo F, Gil J. Informes de Salud Laboral. España 2001-2010 [Internet]. España: CISAL-UPF; 2013 p. 95. Disponible en: http://www.aespla.com/attachments/article/57/2013 02 ISL_2001-2010.pdf 
12. Nussbaum MCN. Las Fronteras de la Justicia. Consideraciones sobre la exclusión. 2012. a ed. España: Espasa Libros; 2012. 447 p.

13. Sen, Amartya. La idea de la justicia. 1a ed. Colombia: Taurus Pensamiento; 2011. 499 p.

14. Sen, Amartya. Bienestar, justicia y mercado. 1 Ed. Barcelona: Ediciones Paidós Ibérica; 1997. 156 p.

15. Reyes A. La proteccion de las personas con discapacidad en la Convencion Americana sobre Derechos Humanos desde el enfoque de las capacidades: de la igualdad de trato a la igualdad de oportunidades. Am Univ Int Law Rev [Internet]. 2015 [citado 4 de mayo de 2017];30:249-284. Disponible en: https:// litigation-essentials.lexisnexis.com/webcd/app?action=DocumentDisplay \&crawlid $=1 \&$ doctype $=$ cite \&docid $=30+$ Am. + U. + Int $\% 271+$ L. + Rev. $+249 \&$ srctype $=$ smi\&srcid $=3$ B $15 \& k e y=$ cfd0f5916a22e 1 b589 435aed408598e 0

16. Strapazzon C, Pinheiro M. Direitos humanos fundamentais das pessoas com deficiências: the capability approach. Rev AJURIS [Internet]. 2014 [citado 14 de octubre de 2017];41(155-183). Disponible en: http://www.ajuris.org.br/OJS2/index.php/REVAJURIS/article/view/224

17. Torres HF, Molina DC. Justicia, igualdad, discapacidad: una reflexión desde el "enfoque de las capacidades" y la teoría de la justicia de John Rawls. Rev Chil Ter Ocupacional [Internet]. 2014 [citado 18 de julio de 2016];14(2):71-82. Disponible en: http://www.revistaestudiosarabes.uchile.cl/index.php/ RTO/article/viewArticle/35711

18. Di Tullio A. ¿ Hacia una justicia sin fronteras? El enfoque de las capacidades de Martha Nussbaum y los límites de la justicia. Daimon Rev Int Filos [Internet]. 2013 [citado 4 de mayo de 2017];(58):5168. Disponible en: http://revistas.um.es/daimon/article/view/144611

19. Reicher SC. Human Diversity and Asymmetries: A Reinterpretation of the Social Contract under the Capabilities Approach. SUR- Int J Hum Rights [Internet]. 2011 [citado 13 de octubre de 2017];14:167-79. Disponible en: http://heinonline.org/hol-cgi-bin/get_pdf.cgi?handle=hein.journals/ surij14\&section $=12$

20. Franco S. Los verbos esenciales de la salud pública. En: Edmundo Granda Ugalde La salud y la vida Varios autores [Internet]. Quito: Organizacion Panamericana de la Salud; 2011 [citado 12 de noviembre de 2017]. p. 142. Disponible en: http://www.observatoriorh.org/sites/default/files/webfiles/fulltext/saludyvida_vol3.pdf

21. Gadamer H. Verdad y Método. Fundamentos de una hermenéutica filosófica. Salamanca: Sígueme; 1988.

22. Honneth, Axel. La lucha por el reconocimiento [Internet]. Crítica (Grijaldo Mondadori S.A). Barcelona; 1997 [citado 16 de octubre de 2015]. 227 p. Disponible en: https://filosinsentido.files.wordpress. com/2013/06/73040182-honneth-a-la-lucha-por-el-reconocimiento.pdf

23. Congreso de la República de Colombia. Ley 361 [Internet]. Diario Oficial 42.978 del 11 de febrero 1997. Disponible en: http://www.alcaldiabogota.gov.co/sisjur/normas/Norma1.jsp?i=343

24. Congreso de la República de Colombia. Ley 1450 [Internet]. Diario Oficial 48.10216 de junio 2011. Disponible en: http://www.alcaldiabogota.gov.co/sisjur/normas/Norma1.jsp?i=43101

25. Congreso de la República de Colombia. Ley 1562 [Internet]. Diario Oficial 48.488 de 7 de julio de 2012 2012. Disponible en: http://wsp.presidencia.gov.co/Normativa/Leyes/Documents/ley156211072012.pdf

26. Congreso de la República de Colombia C. Ley 1566 [Internet]. Diario Oficial 37.852 de 21 de abril 2012. Disponible en: http://wsp.presidencia.gov.co/Normativa/Leyes/Documents/ley156631072012.pdf

27. Congreso de la República de Colombia. Ley 1616 [Internet]. Diario Oficial 48.680 de 21 de enero 2013. Disponible en: http://wsp.presidencia.gov.co/Normativa/Leyes/Documents/2013/LEY\%20 1616\%20DEL\%2021\%20DE\%20ENERO\%20DE\%202013.pdf

28. Congreso de la República de Colombia. Ley 1618 [Internet]. Diario Oficial 48.717 de 27 de febrero de 2013 2013. Disponible en: http://54.226.140.140/men/docs/pdf/ley_1618_2013.pdf 
29. Congreso de la República de Colombia. Ley 1751 [Internet]. Diario Oficial No. 49.427 de 16 de febrero 2015. Disponible en: https://adapt.it/EJCLS/index.php/rlde_adapt/article/view/296

30. Congreso de la República de Colombia. Ley 1753 [Internet]. Diario oficial No 49.538 de 9 de junio de 2015 2015. Disponible en: http://sidegap.com.co/sitio/wp-content/uploads/2015/06/plan nacional desarrollo.pdf

31. Vásquez ML, Ferreira MR, Mogollón A, Fernández MJ, Delgado ME, Vargas I. Introducción a las técnicas cualitativas de investigación aplicadas en salud. Cali: Universidad del Valle; 2011. 172 p.

32. Páramo P. La investigación en ciencias sociales: Estrategias de Investigación. U. Piloto de Colombia; 2011. $329 \mathrm{p}$.

33. Congreso de la República de Colombia. 1438 [Internet]. 2011. Disponible en: http://www.alcaldiabogota.gov.co/sisjur/normas/Norma1.jsp?i $=41355$

34. Hammell KW. Quality of life, participation and occupational rights: A capabilities perspective. Aust Occup Ther J [Internet]. 1 de abril de 2015 [citado 24 de julio de 2016];62(2):78-85. Disponible en: http://onlinelibrary.wiley.com/doi/10.1111/1440-1630.12183/abstract

35. Toboso M, Arnau M. La discapacidad dentro del enfoque de capacidades y funcionamientos de Amartya Sen. Rev Araucaria [Internet]. 2008 [citado 12 de abril de 2015];20:1-39. Disponible en: http:// digital.csic.es/handle/10261/23277

36. Organization WH, others. Informe sobre la salud en el mundo 2001: Salud mental: nuevos conocimientos, nuevas esperanzas. 2001 [citado 4 de octubre de 2017]; Disponible en: http://cdrwww.who. int/entity/whr/2001/en/whr01_es.pdf

37. Duque SP, Gómez NE, Villadiego ÁM. Labor protection for the elderly with physical, mental, or sensory disabilities. Rev Fac Nac Salud Pública [Internet]. 2012 [citado 8 de agosto de 2016];30(2):212-221. Disponible en: http://www.scielo.org.co/scielo.php?pid=S0120-386X2012000200010\&script $=$ sci $\underline{\operatorname{arttext} \& \operatorname{tlng}=\mathrm{es}}$

38. Restrepo O, Diego A, Jaramillo E, Juan C. Concepciones de salud mental en el campo de la salud pública. Rev Fac Nac Salud Pública [Internet]. 2012 [citado 26 de marzo de 2016];30(2):202-211. Disponible en: http://www.scielo.org.co/scielo.php?pid=S0120-386X2012000200009\&script=sci abstract

39. Organización Mundial de la Salud, Banco Mundial. Informe mundial sobre discapacidad [Internet]. Geneva, Switzerland: OMS; 2011 p. 388. Disponible en: http://www.who.int/disabilities/world report/2011/es/

40. Organización Mundial de la Salud. Salud mental: fortalecer nuestra respuesta [Internet]. WHO. [citado 27 de noviembre de 2017]. Disponible en: http://www.who.int/mediacentre/factsheets/fs220/es/

41. Organización Panamericana de la Salud. Salud mental y discapacidad [Internet]. 1a ed. Argentina: OPS; 2009 [citado 27 de noviembre de 2017]. 207 p. Disponible en: http://publicaciones.ops.org.ar/ publicaciones/coleccionOPS/pub/pub65.pdf 